\title{
The Skin and Nose Microbiome and Its Association with Filaggrin Gene Mutations in Pediatric Atopic Dermatitis
}

\author{
Minke M.F. van Mierlo ${ }^{a} \quad$ Luba M. Pardo $^{a}$ Karin B. Fieten $^{b}, c, d \quad$ Tim J. van den Broek $^{e}$ \\ Frank H.J. Schuren ${ }^{\mathrm{e}}$ Michel van Geel ${ }^{\mathrm{f}, \mathrm{g}}$ Suzanne G.M.A. Pasmans ${ }^{\mathrm{a}}$ \\ aDepartment of Dermatology-Center of Pediatric Dermatology, Sophia Children's Hospital, Erasmus MC University \\ Medical Center Rotterdam, Rotterdam, The Netherlands; bepartment of Dermatology and Allergology, University \\ Medical Center, Utrecht, The Netherlands; ' Dutch Asthma Center Davos, Davos, Switzerland; dSwiss Institute of Allergy \\ and Asthma Research (SIAF), University of Zurich, Davos, Switzerland; 'Microbiology and Systems Biology, TNO Zeist, \\ Zeist, The Netherlands; ${ }^{f}$ Department of Dermatology, Maastricht University Medical Centre, Maastricht, The Netherlands; \\ gDepartment of Clinical Genetics, Maastricht University Medical Centre, Maastricht, The Netherlands
}

\section{Keywords}

Eczema · Filaggrin - Microbiome - Skin barrier ·

Staphylococcus aureus

\begin{abstract}
Background: Interactions between the skin barrier, immune system, and microbiome underlie the development of atopic dermatitis (AD). Objective: To investigate the skin and nasal microbiome in relation to filaggrin gene $(F L G)$ mutations. Methods: A cross-sectional study including 77 children with difficult-to-treat $\mathrm{AD}$. The entire encoding region of $F L G$ was screened for mutations using single molecule molecular inversion probes and next-generation sequencing. Bacterial swabs from the anterior nares, lesional and nonlesional skin were analyzed using 16 S rRNA sequencing. For skin samples, additional qPCR was performed for Staphylococcus aureus and Staphylococcus epidermidis. Results: The prevalence of patients with a mutation in $F L G$ was $40 \%$, including 10 different mutations. Analyzing bacterial swabs from all three niches showed a significant effect for both niche and FLG mutation status on the overall microbiome composition. Using a subset analysis to test the effect of $F L G$ mutation status per
\end{abstract}

karger@karger.com www.karger.com/drm

Karger $\stackrel{\text { ' }}{5}$

BOPEN ACCESS
(C) 2022 The Author(s)

Published by S. Karger AG, Basel

This is an Open Access article licensed under the Creative Commons Attribution-NonCommercial-4.0 International License (CC BY-NC) (http://www.karger.com/Services/OpenAccessLicense), applicable to the online version of the article only. Usage and distribution for commercial purposes requires written permission. niche separately did not show a significant association to the microbiome. Shannon diversity and S. aureus abundance were significantly affected by the niche, but not by the presence of an FLG mutation. Conclusions: Our results suggest only a minor role for FLG mutation status on the overall microbiome, which is rather caused by differences in the present genera than by microbe richness and evenness.

(c) 2022 The Author(s)

Published by S. Karger AG, Basel

\section{Introduction}

Atopic dermatitis $(\mathrm{AD})$ is a chronic inflammatory skin disorder primarily present in children [1]. This condition is characterized by a dry, itchy skin with eczematous skin lesions that show an age-related distribution [2]. The pathogenesis of $\mathrm{AD}$ is complex, including alterations in the skin microbiome, epidermal barrier dysfunction, and immunological changes $[1,3]$.

The skin microbiome in $\mathrm{AD}$ is characterized by a low bacterial diversity, with a high abundance of Staphylococcus

Luba M. Pardo and Karin B. Fieten contributed equally to this work.
Correspondence to:

Minke M.F. van Mierlo, m.vanmierlo@erasmusmc.n Suzanne G.M.A. Pasmans, s.pasmans@erasmusmc.nl 
aureus and Staphylococcus epidermidis and low abundance of the genera Propionibacterium, Streptococcus, Acinetobacter, Corynebacterium, and Prevotella [4]. The skin microbiome is in constant interaction with the skin barrier and immune system, leading to inflammation characteristic of AD [5-7]. Previous studies showed that skin barrier impairment, characterized by mutations in the filaggrin gene $(F L G)$, causes modified fibronectin binding, higher skin $\mathrm{pH}$, and an increased serine protease activity, which can lead to altered colonization patterns $[8,9]$. Interestingly, skin colonization with $S$. aureus leads to the secretion of virulence factors, increasing inflammation and further damage of the skin barrier $[8,10]$. Furthermore, activated Thelper type 2 (Th2) cells in AD can downregulate the filaggrin expression in the stratum corneum (SC) [11, 12].

Mutations in FLG are the strongest genetic risk factors for $\mathrm{AD}$ susceptibility $[11,13]$. In West-European populations, 2282del4, R2447*, R501*, and S3247* are the most common mutations with a prevalence of around $40 \%$ in $\mathrm{AD}$ patients $[11,14]$. However, up to 113 different mutations have been described in the literature [15], which are present depending on ethnic background $[16,17]$. Due to the complexity of FLG, with the third exon encoding between 10 and 12 nearly identical repeats, screening for mutations in this gene has been challenging. Classical Sanger sequencing is frequently used, but this method is too cumbersome to screen the whole encoding gene for mutations [18]. A recent method, using single molecule molecular inversion probes (smMIPs) and next-generation sequencing (NGS) permits comprehensive sequencing of the entire gene $[15,19]$. Instead of screening for population-specific mutations only, this technique enables to analyze the entire gene and detect population-specific mutations as well as specific mutations not previously published.

Previous studies investigating whether $S$. aureus colonization is more common in patients with a mutation in FLG compared to wild-type patients, show conflicting results $[10,20-23]$. Publications on the relation between mutations in FLG and the composition of the entire microbiome in $\mathrm{AD}$ are scarce. It was recently shown that the microbiome on nonlesional skin in adult AD patients was associated with FLG mutation status [7]. This study included a smaller number of $\mathrm{AD}$ patients and only screened for the most common mutations in FLG, which might have led to an underrepresentation of FLG mutation carriers. In addition, no other studies are available to validate these results.

The aim of this study was to profile the entire mutational background of FLG using a new smMIP sequencing technique in children with difficult-to-treat AD. Second, we describe the microbiome in this population and investigate differences in the microbiome from nose, lesional and nonlesional skin in $\mathrm{AD}$ children with and without a mutation in FLG.

\section{Material and Methods}

Study Design

This study was performed as part of the DAVOS-trial, a pragmatic randomized controlled trial (RCT) comparing the longterm effectiveness of treatment in alpine climate to treatment in moderate maritime climate in children with difficult-to-treat $\mathrm{AD}$ (ISRCTN88136485). The detailed study protocol and primary outcomes have been published previously [24,25]. Study procedures were reviewed and approved by the Medical Ethics Committee of the University Medical Center Utrecht, the Netherlands (reference 09-192/K). All patients and, if needed, their parents/guardians provided written informed consent. This present study involves secondary outcomes of this trial and included data before the start of the intervention period.

\section{Participants}

This study included Dutch children between 8 and 18 years old, with difficult-to-treat $\mathrm{AD}$. Difficult-to-treat $\mathrm{AD}$ was determined before the start of the study and defined as use of at least a class 3 topical corticosteroid and not being able to step down, current use of systemic immunosuppressive treatment, repeated treatment with potent topical corticosteroids or systemic immunosuppressive treatment, a history of use of systemic treatment, a significant impact of $\mathrm{AD}$ on the child's or the family's quality of life, or seemingly unresponsive to conventional therapy according to current guidelines [25]. Demographic data was extracted from questionnaires and the electronic patient files. Due to the severity of the disease in this patient group, concurrent medication was continued. Disease severity was determined using the Self-Administered Eczema Area and Severity Index (SA-EASI, range 0-96), and microbiome as well as blood samples were collected. Study assessments were performed in the Netherlands.

\section{Microbial Samples}

Microbial samples were collected from the anterior nares, lesional and nonlesional skin. Samples taken from the lesional skin were preferably taken from the antecubital fold or the popliteal fold. Nonlesional skin samples were taken from the volar arm if possible (online suppl. Table 1; for all online suppl. material, see www.karger.com/doi/10.1159/000520978) [26]. All swabs were collected using sterile cotton swabs soaked in sterile $\mathrm{NaCl} 0.9 \%$ and stored at $-80^{\circ} \mathrm{C}$ until further processing.

\section{DNA Isolation, $q P C R$, and Sequencing}

Extensive description of the procedures have been published previously [26]. Microbiome analysis was performed on bacterial swabs from the nasal cavity, lesional and nonlesional skin with sequencing of the $16 \mathrm{~S}$ rRNA gene using V4 hypervariable region on the Illumina MiSeq sequencer (Illumina, San Diego, CA, USA). A genus table with raw read counts was generated for downstream analysis. Quantitative (q)PCR was performed on samples from the lesional and nonlesional skin to identify S. aureus and S. epidermidis. The bacterial DNA concentration was reported as $\log 10$-transformed femtogram per microliter $(\mathrm{fg} / \mu \mathrm{L})$ in this paper. 
Table 1. Patient characteristics

\begin{tabular}{|c|c|c|c|}
\hline & $\begin{array}{l}\text { Wild-type } \\
(n=46)\end{array}$ & $\begin{array}{l}\text { FLG mutation } \\
\text { carriers }^{\mathrm{a}}(n=31)\end{array}$ & $p$ value $^{b}$ \\
\hline Sex (female), $n(\%)$ & $20(43.5)$ & $19(61.3)$ & 0.13 \\
\hline Age, median (IQR) & $13.0(11.0-15.0)$ & $12.0(11.0-15.0)$ & 0.64 \\
\hline Age of AD onset $<6$ months, $n(\%)$ & $34(80.4)$ & $28(90.3)$ & 0.34 \\
\hline Asthmac, $n(\%)$ & $39(84.8)$ & $26(83.9)$ & 0.91 \\
\hline Rhinitis ${ }^{\mathrm{d}}, n(\%)$ & $38(82.6)$ & $29(93.5)$ & 0.30 \\
\hline Food allergy ${ }^{\mathrm{e}, \mathrm{f}}, n(\%)$ & $25(54.3)$ & $26(83.9)$ & $<0.01$ \\
\hline SA-EASI' ${ }^{9}$, median (IQR) & $37.2(17.6-52.6)$ & $41.4(26.4-60.6)^{\mathrm{h}}$ & 0.29 \\
\hline Topical corticosteroids previous 7 daysi,$n$ (\%) & & & 0.89 \\
\hline None & $1(2.2)$ & $1(3.2)$ & \\
\hline Moderate & $2(4.3)$ & $2(6.5)$ & \\
\hline Potent & $40(87.0)$ & $27(87.1)$ & \\
\hline Very potent & $3(6.5)$ & $1(3.2)$ & \\
\hline Systemic medication, $n(\%)$ & & & 1.00 \\
\hline Prednisone & $2(4.3)$ & $1(3.2)$ & \\
\hline Cyclosporine & $4(8.7)$ & $3(9.7)$ & \\
\hline Oral antibiotics & - & $1(3.2)$ & \\
\hline
\end{tabular}

$A D$, atopic dermatitis; FLG, filaggrin gene; SA-EASI, Self-Administered Eczema Area and Severity Index; SD, standard deviation. ${ }^{a}$ Including 23 patients who were heterozygous, 4 patients homozygous, and 4 patients with two different mutations. ${ }^{b} p$ values were analyzed using $x^{2}$, Fisher's exact test, or Mann-Whitney $U$ when appropriate. ${ }^{C}$ Asthma was diagnosed based on spirometry reversibility testing and Methacholine Challenge Test. ${ }^{d}$ Rhinitis was diagnosed based on assessment by a pediatrician. ${ }^{e}$ Food allergy was defined as a positive double-blind placebo-controlled food challenge (DBPCFC) or convincing clinical history (a reported type I allergic reaction with acute symptoms within $2 \mathrm{~h}$ after ingestion of the food) in combination with sensitization to the specific food allergen. ${ }^{f}$ Missing $n=2$ (both groups $n=1$ ). ${ }^{\text {gRange }}$ 0-96 ( $<17$ mild; $18-46$ moderate; $>47$ severe $)^{1}$. ' ${ }^{\text {M Missing }} n=1$. 'UK potency system used.

\section{FLG Mutation Profiling}

FLG mutations were determined on DNA isolated from blood samples. We used smMIP-NGS strategy to screen the entire FLG and to identify all mutations resulting in premature protein termination. Detailed information about the sample preparation and smMIP-NGS technique have been described previously $[15,19]$. Previous use of this technique showed that more than one mutation may be present on the same allele, especially when this includes rare or new mutations. Therefore, patients in which we found two different mutations in FLG were not classified as compound heterozygous but as patients with more than one mutation. "FLG mutation status" was used throughout this paper to describe the dichotomous outcome as used in our statistical models: FLG wild-type patients $\left(F L G^{+}\right)$versus $F L G$ mutation carriers $\left(F L G^{-}\right.$, patients with one or more mutations in $F L G$ ).

\section{Statistical Analysis}

To determine differences in demographic data and disease severity between patients with and without a mutation in FLG, $\chi^{2}$, Fisher's exact test, or Mann-Whitney U tests were used when appropriate. Descriptive statistics were used to present the FLG mutations in our study population. For analysis of the microbiome, we used non-subsampled genus tables after filtering (Shannon diversity index was calculated on unfiltered data). Filtering was applied by selecting the genera representing $97.5 \%$ of all counts. Prior to ordination analysis, the filtered genus tables were square-root transformed with subsequent application of Wisconsin double standardization. To visualize the microbial composition for all 3 niches sampled (nose, lesional and nonlesional skin) and FLG status $\left(F L G^{+}\right.$and $\left.F L G^{-}\right)$, Bray-Curtis distance-based multidimensional scaling (MDS) was used. Permutational multivariable analysis of variance (PERMANOVA) correcting for repeated measurements was used to determine significant differences in microbial composition while accounting for FLG mutation status. We included the covariates "niche" and "mutation status" as interaction terms in this model. When appropriate, PERMANOVA coefficients were obtained to determine the genera that contributed to the significant differences [27]. Second, we performed a subset analysis for the skin samples and the niches individually (nose, lesional and nonlesional skin) to investigate the effect of FLG mutation status on the microbial composition within each niche.

In-depth analysis was performed to assess the effect of FLG mutation status on $S$. aureus and S. epidermidis colonization rates using a $\chi^{2}$ test. Furthermore, the effect on S. aureus and S. epidermidis abundance in patients with positive bacterial swabs was analyzed with a linear mixed-effect model. This statistical model was also used in analyzing the effect of FLG status on the Shannon diversity index.

Statistical analyses were performed in SPSS (version 21) and R software (version 3.5.1). We used the packages "phyloseq" and "vegan" for microbiome analyses and accounted for repeated measurements using the "strata" argument [28]. R package "ggplot2" was used for visualization [29]. Linear mixed-effect models were performed using "lme" and "lme4" package [30]. A $p$ value of $\leq 0.05$ was considered statistically significant. 

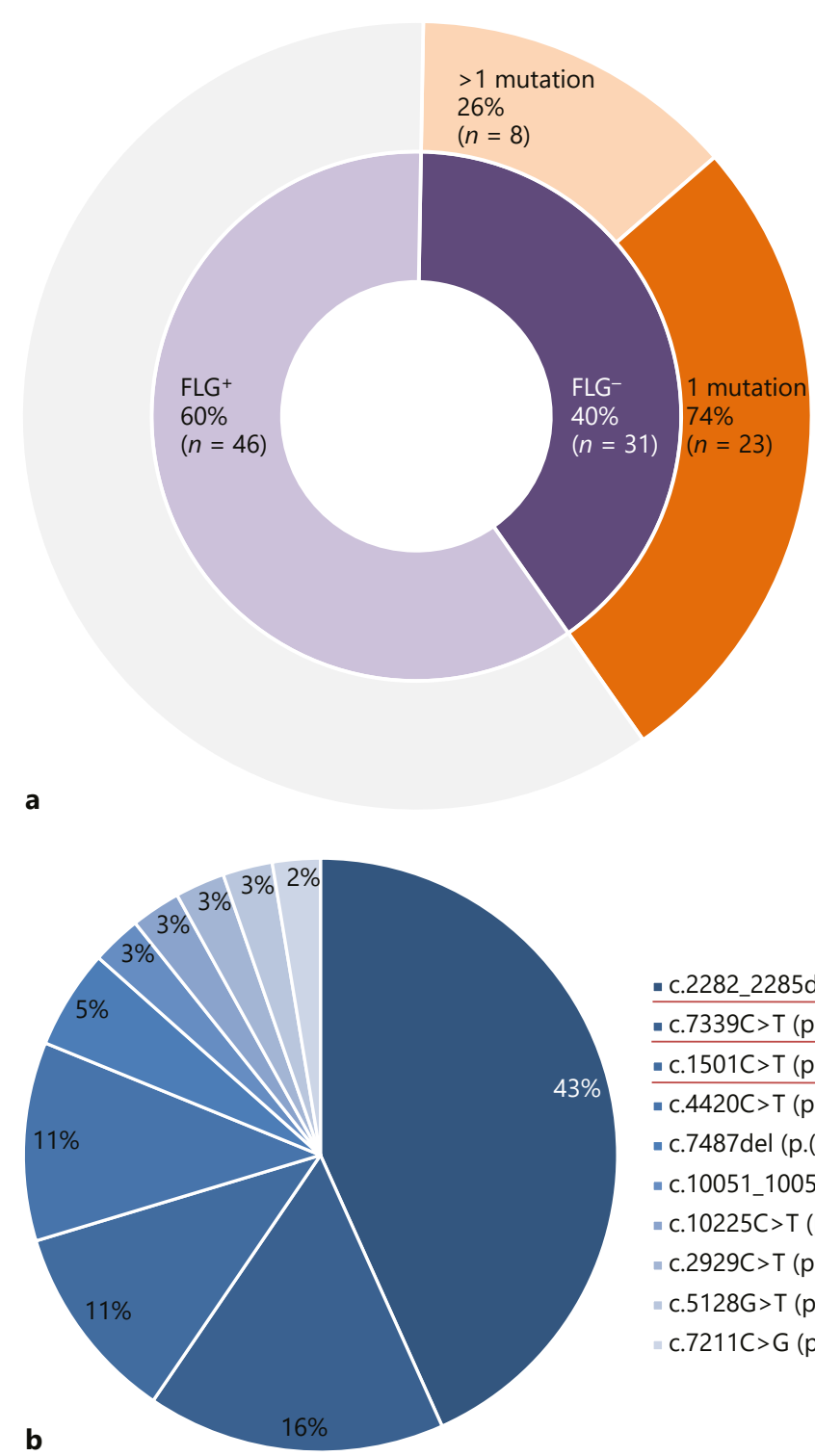

- c.2282_2285del (p.(Ser761Cysfs*36))

- c.7339C >T (p. $(R 2447 *))$

- c.1501C>T (p.(Arg501*))

- c.4420C > T (p.(Arg1474*))

- c.7487del (p.(Thr2496Asnfs*104))

- c.10051_10054del

= c.10225C>T (p.(Arg3409*))

- c.2929C>T (p. (Gln977*))

« c.5128G > T (p.(Gly1710*))

- c.7211C>G (p.(Ser2404*))

Fig. 1. Prevalence of FLG mutations using smMIP sequencing. a Prevalence of patients carrying FLG mutations. $40 \%(n=31)$ of the patients had a mutation in FLG. Within this group of mutation carriers, more than one mutation was detected in 8 (26\%) patients. b Prevalence of the 10 different FLG mutations in our study population. Underlined mutations represent mutations which are most commonly detected in West-European populations.

\section{Results}

\section{Study Subjects and Sample Characteristics}

A total of 77 patients were included in the study. Lesional and nonlesional skin swabs were available for 76 patients (99\%) and analyzed using qPCR techniques. Sufficient amount of DNA to perform $16 \mathrm{~S}$ rRNA sequencing was available in $63(82 \%)$ patients for lesional skin, 55 (71\%) patients for nonlesional skin, and 73 (95\%) for the nose (online suppl. Fig. 1). Patient characteristics of missing data did not differ from the study group. Demographic data, comorbidities, medication use, and disease severity are noted in Table 1 . The median severity score was 37.2 (17.6-52.6) in the $F L G^{+}$group, compared to 41.4 (26.4-60.6) in the $F L G^{-}$group $(p=0.29)$. 


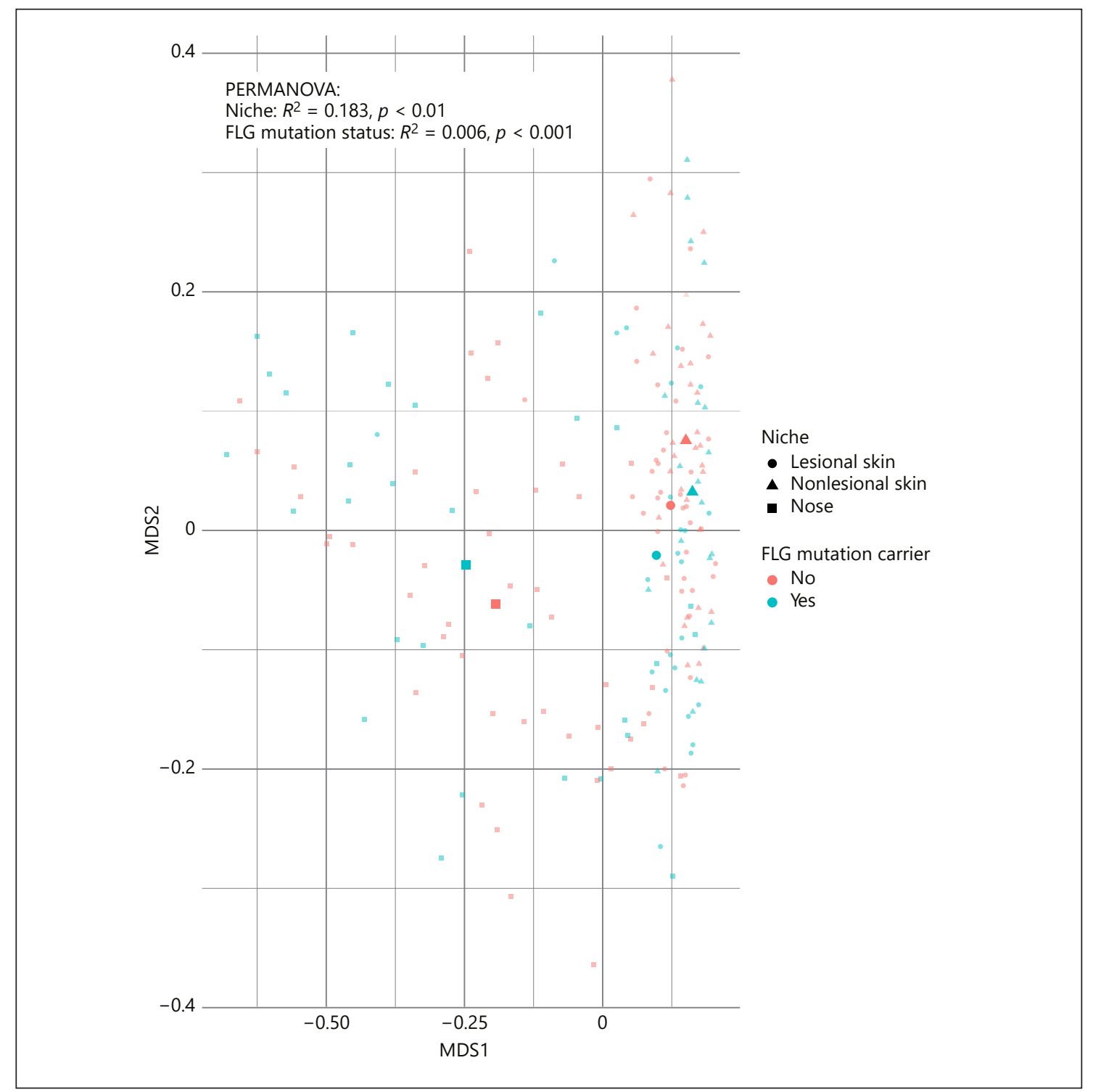

Fig. 2. Bray-Curtis distance-based multidimensional scaling plot showing the microbiota for nose and skin in relation to FLG mutation status. For ordination analysis, genus tables were standardized using square-root transformation with subsequent application of

Screening the Entire Filaggrin Gene Improves the Sensitivity of Mutation Detection

A total of 10 different mutations leading to a premature protein termination were found, of which c. $5128 \mathrm{G}>\mathrm{T}$ (p. $($ Gly1710*)) in repeat 4 and c.7211C $>\mathrm{G}$ (p. $\left(\right.$ Ser $\left.\left.2404^{*}\right)\right)$ in repeat 6 have not been described before (Fig. 1, online suppl. Table 2). Compared to the most common mutations in West-European populations (2282del4, R2447*, R501*, and S3247*), the prevalence of patients with an FLG mutation increased from
Wisconsin double standardization. FLG status was significantly associated with the overall microbial composition using PERMANOVA analysis (niche: $R^{2}=0.183, p<0.01$; FLG status: $R^{2}=$ $0.006, p<0.01$; interaction: $R^{2}=0.008, p=0.47$ ).

$33 \%$ ( 25 of 77 ) to $40 \%$ ( 31 of 77 ) screening the entire encoding gene. This included $8(26 \%)$ patients with more than one mutation.

\section{The Microbial Composition in Children with $A D$}

All sequences belonged to 844 genera, of which 81 genera remained after filtering. The genus Staphylococcus was predominant in both lesional and nonlesional skin samples, with a median relative abundance (RA) of $78 \%$ and $44 \%$, respectively. This was followed by Streptococcus 
Fig. 3. Shannon diversity index in relation to FLG mutation status. Shannon diversity index represents the number of different genera (richness) and how even they are distributed (evenness). The boxes represent the twenty-fifth percentile, median, and seventy-fifth percentile. FLG status did not affect the microbial composition in all three niches as determined using a linear mixed-effect model (niche $p=0.01 ; F L G$ status $p=0.35$; "niche: FLG status" $p=$ $0.85)$.

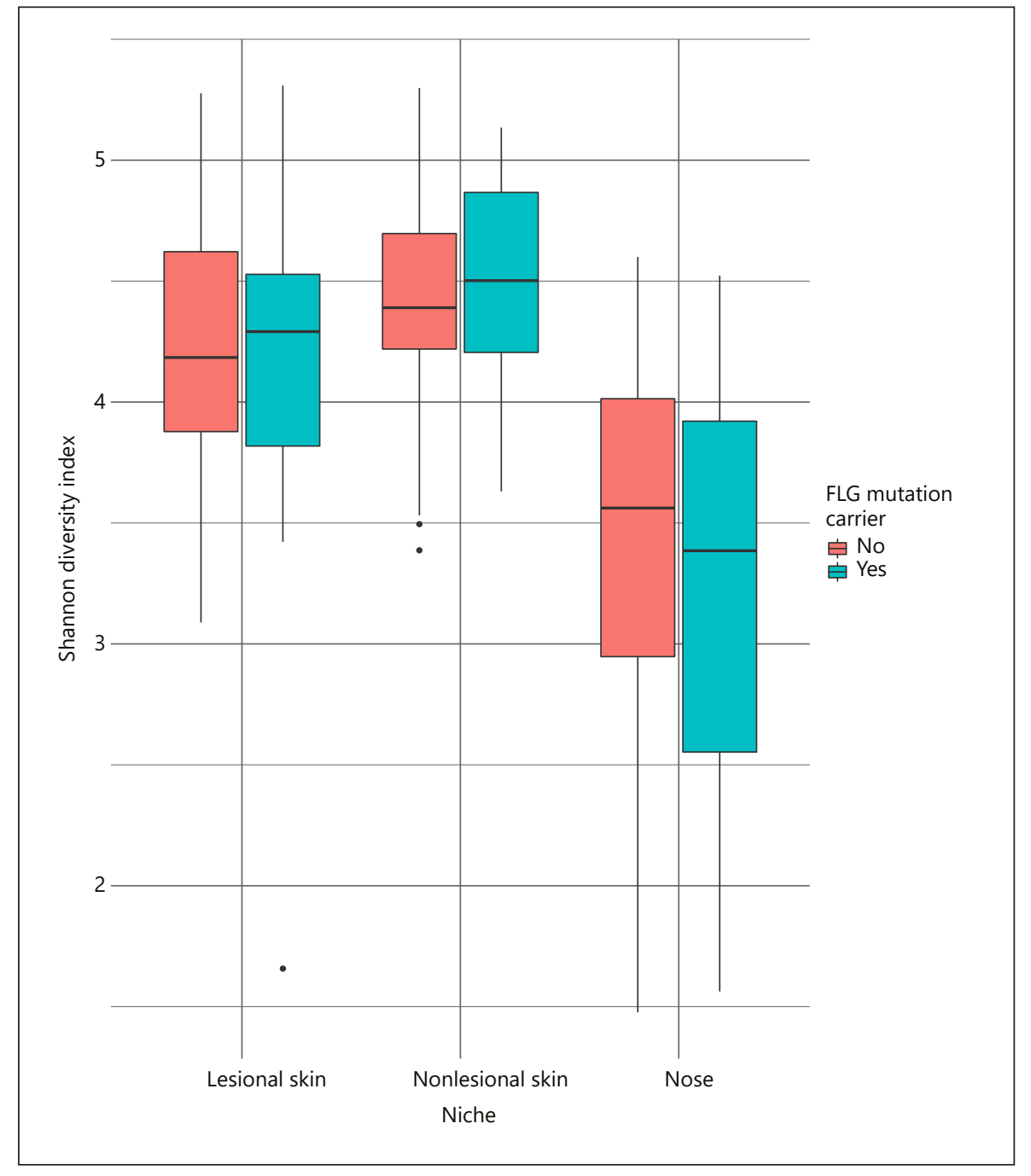

(median RA 3\% and 4\%) and Corynebacterium genera (median RA 2\% and 5\%) (online suppl. Fig. 2). Within the nose, the genera Corynebacterium (31\%), Staphylococcus (11\%), and Dolosigranulum genera (9\%) were most prevalent (online suppl. Fig. 2). The differences in microbial composition between the three sampled niches were visualized and tested (online suppl. Fig. 3). All three niches harbored a significantly different microbiome (lesional skin vs. nonlesional skin, nose vs. lesional skin, nose vs. nonlesional skin: PERMANOVA $R^{2}=0.027, p<0.01$; $R^{2}=0.190, p<0.01$; and $R^{2}=0.139, p<0.01$, respectively).

\section{The Overall Microbial Composition Is Affected by FLG Mutation Status}

Community composition was visualized considering FLG mutation status in Figure 2 (skin samples only in online suppl. Fig. 4). The PERMANOVA analysis showed that both niche and FLG mutation status were significantly associated with the overall microbial composition (niche: $R^{2}=0.183, p<0.01$; FLG status: $R^{2}=0.006, p<$ 0.01 ; interaction "niche: FLG status": $R^{2}=0.008, p=0.47$ ). The association between the microbiome and FLG mutation status was mainly driven by the Corynebacterium, which was less abundant in FLG mutation carriers (online suppl. Fig. 5a). Significant effects for niche and FLG mutation status were also observed in the subset analysis including only skin samples (niche: $R^{2}=0.027, p<0.01$; FLG status: $R^{2}=0.010, p<0.01$; interaction "niche: FLG status": $R^{2}=0.005, p=0.56$; online suppl. Fig. 4 ). In this model, the Staphylococcus (less abundant in $F L G^{-}$) and Acidovorax genera (more abundant in $F L G^{-}$) mainly contributed to the effect for FLG mutation status (online suppl. Fig. 5b). Performing subset analysis to test the effect of FLG status within each niche separately (nose, lesional 
Fig. 4. Quantitative PCR results of the skin samples for $S$. aureus and $S$. epidermidis in relation to FLG mutation status. The results for $S$. aureus and S. epidermidis load are shown using $\log 10$ transformed data. The boxes represent the twenty-fifth percentile, median, and seventy-fifth percentile. $S$. aureus density was significantly associated with the sampled niche, but not with FLG status (niche $p<0.01 ;$ FLG status $p=0.10)$.

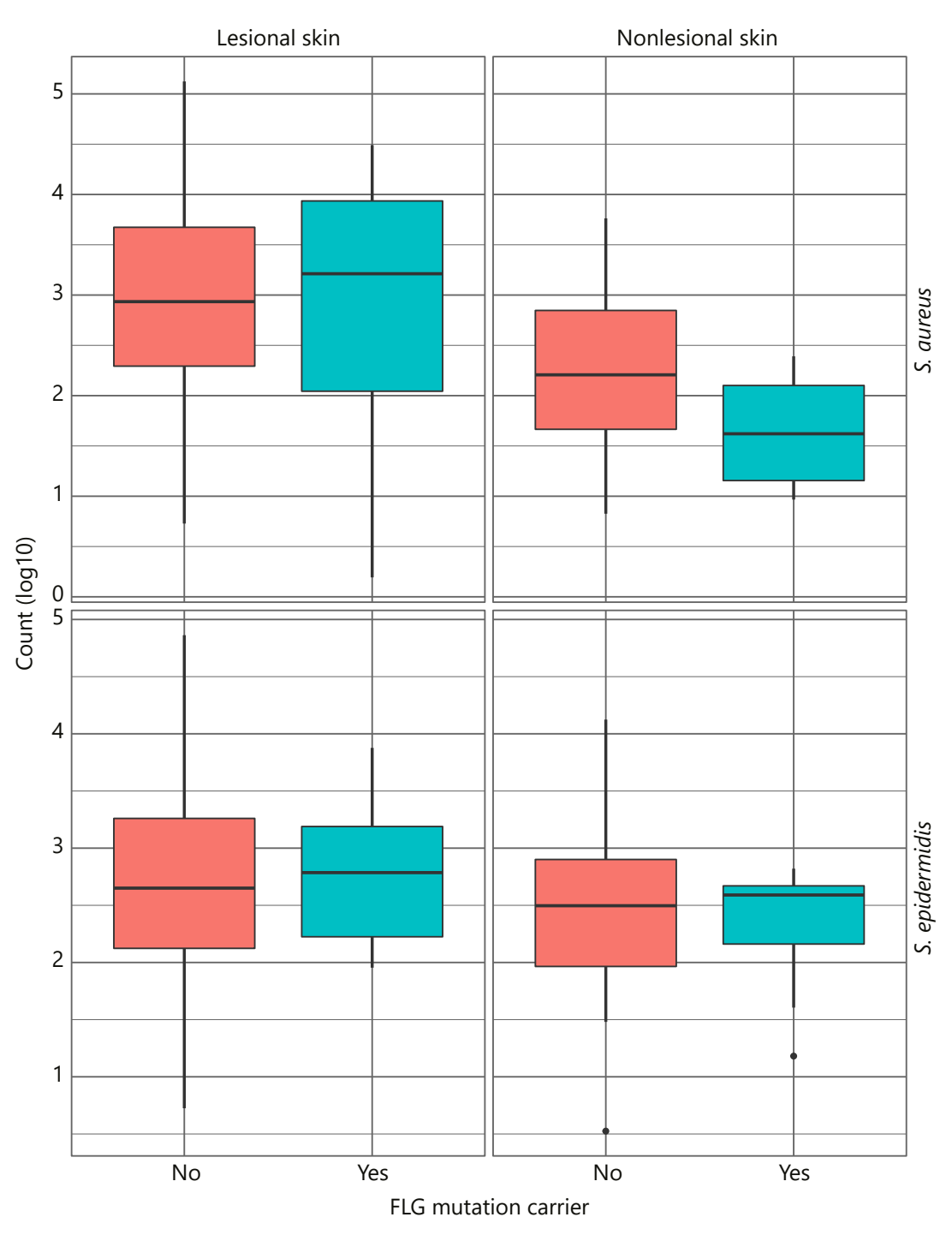

Table 2. Colonization rates for S. aureus and S. epidermidis

\begin{tabular}{|c|c|c|c|}
\hline & $\begin{array}{l}\text { Wild-type } \\
(n=46)\end{array}$ & $\begin{array}{l}\text { FLG mutation } \\
\text { carriers }(n=31)\end{array}$ & $\begin{array}{l}p \\
\text { value }^{a}\end{array}$ \\
\hline \multicolumn{4}{|l|}{ Positive for S. aureus } \\
\hline Lesional skin & $37(80.4)^{\mathrm{b}}$ & $19(61.3)^{c}$ & 0.10 \\
\hline Nonlesional skin & $26(56.5)^{c}$ & $10(32.3)^{c}$ & 0.04 \\
\hline \multicolumn{4}{|c|}{ Positive for S. epidermidis } \\
\hline Lesional skin & $43(93.5)$ & $28(90.3)^{b}$ & 0.65 \\
\hline Nonlesional skin & $43(93.5)$ & $29(93.5)^{b}$ & 0.54 \\
\hline
\end{tabular}

$F L G$, filaggrin gene. ${ }^{a} p$ values were analyzed using $X^{2}$. Number of missings: ${ }^{\mathrm{b}} n=1 ;{ }^{\mathrm{c}} n=2$. and nonlesional skin) did not yield any significant associations between FLG status and microbiome composition (nose PERMANOVA $R^{2}=0.014, p=0.39$; lesional skin PERMANOVA $R^{2}=0.013, p=0.72$; nonlesional skin PERMANOVA $R^{2}=0.0185, p=0.42$ ). Alpha diversity, indicated with Shannon diversity index, was significantly affected by niche $(p=0.01)$ but not by FLG status ( $p=$ 0.35 , interaction "niche: FLG status" $p=0.85$ ) (Fig. 3).

\section{Association between Staphylococcus Abundance and FLG Status}

qPCR was performed to gain more information on the species $S$. aureus and S. epidermidis. In the total study population, $56(72 \%)$ patients were positive for $S$. aureus 
on lesional skin, with similar proportions in $\mathrm{FLG}^{+}$and $F L G^{-}$patients $(p=0.10)$ (Table 2). Interestingly, on nonlesional skin, we found a higher proportion of patients colonized with $S$. aureus in the $F L G^{+}$group than in the $F L G^{-}$group ( $57 \%$ for $F L G^{+}$vs. $32 \%$ in $F L G^{-}$group; $p=$ 0.04 ) (Table 2). The mixed model analysis showed that niche (lesional, nonlesional) was significantly associated with $S$. aureus load, but not with FLG mutation status (niche $p<0.01$; FLG status $p=0.10$; interaction "niche: FLG status" $p=0.81$ ) (Fig. 4). S. epidermidis was detected on lesional skin in 70 (91\%) participants and in 69 (90\%) participants on nonlesional skin (Table 2). The S. epidermidis load was not affected by niche $(p=0.17)$, nor by FLG mutation status ( $p=0.08$ ) (Fig. 4).

\section{Discussion}

To our knowledge, this is the first study investigating the effect of FLG mutation status on the microbial composition in children with AD. In this cohort of children with difficult-to-treat $\mathrm{AD}$, the prevalence of FLG mutations was $40 \%$ when screening the entire encoding gene, which is consistent with the prevalence of FLG mutations in $\mathrm{AD}$ groups. We demonstrated that the nose, lesional and nonlesional skin all harbored a significantly different microbiome. In addition, analyzing all samples together (and skin samples only), FLG status was of significant influence on the microbial composition, although the impact on the contribution was rather small. FLG mutations did not affect the Shannon diversity index or $S$. aureus load. Using subset analysis per niche, we were not able to confirm these results for the effect of FLG mutation status on the microbial composition.

The prevalence of FLG mutations was increased with $7 \%$ using the smMIP-NGS strategy in comparison to including only the four most common mutations in the West-European population. It has been shown previously that only analyzing population-specific mutations gives an underrepresentation of FLG mutation prevalence in AD populations [15-17]. Since the C-terminus has a critical role in the processing from profilaggrin to filaggrin and thus the presence of filaggrin in the epidermis [16, 31], all mutations leading to a premature protein termination (and lack of this C-terminal) contribute to the disease burden in $\mathrm{AD}[16,32]$. This underlines the relevance of screening the entire coding region of the $F L G$, instead of focusing on population-specific mutations. Interestingly, the S3247* mutation, which is often

Impact of Filaggrin Mutation Status on the Microbiome described as a common European mutation variant, was not detected in our population. Possible explanations for the absence of S3247* is that mutation distribution can vary in every European country. Second, S3247* is described as a less common variant in comparison to R501* and 2282del4 [16].

A significant effect for FLG mutation status on the overall skin microbiome was found when analyzing all samples together (and analyzing lesional and nonlesional skin samples together), but not using subset analysis per individual niche. This might indicate that the effect for FLG mutation status was only minor $\left(R^{2}\right.$ of 0.006 and 0.010 , respectively) and/or that the number of samples per niche in our study was too small, leading to insufficient power to properly detect significant differences. Only one previous publication, including a smaller number of adult $\mathrm{AD}$ patients with less severe disease, only screening for four mutations in FLG, investigated the association between FLG mutations and the microbiome [7]. They found a significant effect of FLG mutation status on the microbial composition on nonlesional skin (with Staphylococcus caprae being more prevalent in $F L G^{-}$), but not on lesional skin or in the nose [7]. Combining these and our results, a role for FLG status on the microbial composition is suggested. Interestingly, looking into the Shannon diversity index, our results are in line with two previous publications including $\mathrm{AD}$ and ichthyosis vulgaris patients $[7,33]$, showing that Shannon diversity index was not affected by FLG status. The explanation could be that patients with and without a mutation in FLG are colonized by different genera and do not differ based on the number of genera (richness) or how even they are distributed (evenness).

We found similar S. aureus colonization rates in $\mathrm{FLG}^{+}$ and $F L G^{-}$patients on lesional skin, but significantly higher colonization rates on nonlesional skin for $\mathrm{FLG}^{+}$patients. Literature on this association is conflicting. In vitro studies showed that the absence of filaggrin breakdown products, natural moisturizing factor (NMF), increases $S$. aureus corneocyte adhesion and growth rate [20,34]. These findings were supported by clinical data in adult $\mathrm{AD}$ patients, with higher colonization rates on lesional skin and in the nose of patients with a mutation in FLG [21]. In contrast, other clinical studies did not show reduced filaggrin levels in patients colonized with $S$. aureus $[23,35,36]$. The differences in outcomes between clinical studies can be largely affected by disease severity in the studied $\mathrm{AD}$ population. In active $\mathrm{AD}$ skin, Th2 response is upregulated along with the production of cytokines in- 
terleukin (IL)-4 and IL-13, which are known to downregulate filaggrin expression independent of mutation status $[11,12]$. Since our study included patients who were difficult to treat and included $74 \%$ with severe or very severe disease (based on SA-EASI score), it is possible that due to an upregulation of the immune system, filaggrin expression was suppressed irrespective of $F L G$ mutation status and masked the effect for wild-type patients.

A major strength of this study is the comprehensive sequencing of the entire encoding region of FLG, enabling to detect all mutations leading to premature protein termination. This technique enables to identify all patients who are at risk to develop a more severe and persistent disease course and are susceptible for the development of other atopic diseases (i.e., asthma and allergic sensitization). Furthermore, it could be useful to stratify patients in cohort studies. The following limitations need to be mentioned. First, since our cohort included children with difficult-to-treat $\mathrm{AD}$, we did not discontinue concurrent medication. In addition, previous studies have shown that both age (Tanner stage) and disease severity can influence the composition of the microbiome $[37,38]$. In this explorative study, we did not correct for these effects in our statistical analysis. However, the use of topical and systemic medication, age, and disease severity did not differ between patients with and without a mutation in FLG. Furthermore, excluding patients with systemic treatment from our statistical analysis did not yield different results (data not shown). Larger groups of patients with AD might be helpful in correcting for these effects. Second, heterozygous FLG mutations lead to $50 \%$ reduction in filaggrin expression as compared to a complete absence of filaggrin with bi-allelic mutations and might lead to different effects [11]. This gene-dosage effect was suggested in a previous study including ichthyosis vulgaris patients, with significant differences in the microbial composition between bi-allelic mutation carriers and wild-type patients, but not between bi-allelic and heterozygous mutation carriers [33]. Although our study included a small group of patients with more than one mutation in FLG $(n=8)$, we did not find different results for patients with one and more than one mutation in FLG (data not shown). Future studies should include a larger number of patients, investigating the gene-dosage effect on the microbiome in $\mathrm{AD}$. In addition, by measuring FLG breakdown products we can determine the effect of the Th2 response in active AD. Lastly, as described previously [26], the impact of the inability to properly detect the Propionibacterium or to classify the Staphylococci at species level with the use of the V4 hypervariable region, was only minor due to the sampled body regions and the use of qPCR to detect $S$. aureus and S. epidermidis species [39].

In conclusion, sequencing the entire FLG for novel, family- and population-specific mutations, contributing to the disease burden in $\mathrm{AD}$, leads to better patient stratification. Our results suggest that the presence of a mutation in FLG has only a small effect on the microbial composition. Our results indicate that the difference in the microbiome between $F L G^{+}$and $F L G^{-}$patients is rather caused by the presence of distinct genera than differences in the alpha diversity. More research into the interaction of the microbiome with the skin barrier and immune system in large patient groups will lead to a better understanding of the etiology of $\mathrm{AD}$ and will help develop preventive and treatment strategies.

\section{Key Message}

Mutations in the filaggrin gene only have a small impact on the skin microbiome in patients with atopic dermatitis.

\section{Acknowledgement}

This project is part of the European Reference Network (ERN)SKIN. The authors are very grateful to the children and their parents for participating in the study, to J. Beutler for assistance in collection of the samples and Renske Janssen for her technical assistance. We thank the Dutch patient support group VMCE for endorsing the study. We want to acknowledge all dermatologists for enabling patients to participate in our study by referring them to our hospital.

\section{Statement of Ethics}

This research was conducted ethically in accordance with the World Medical Association Declaration of Helsinki. Study procedures were reviewed and approved by the Medical Ethics Committee of the University Medical Center Utrecht, the Netherlands (reference 09-192/K). All patients and, if needed, their parents/guardians provided written informed consent.

\section{Conflict of Interest Statement}

The authors have no conflicts of interest to declare. 


\section{Funding Sources}

This study was supported by unrestricted grants from the European Allergy and Asthma Center Davos, the Merem Dutch Asthma Center Davos, the patient support group "Vereniging Nederland Davos," and Micreos Human Health, the Netherlands.

\section{Author Contributions}

MMFvM, LMP, KBF, and SGMAP designed the study. SGMAP and $\mathrm{KBF}$ designed and supervised the collection of the data. FHJS supervised the DNA isolation, qPCR and sequencing. MvG super- vised the FLG mutation analysis. MMFvM and TJvdB had full access to all of the data and performed the data analyses. MMFvM, $\mathrm{LMP}, \mathrm{KBF}$, and SGMAP interpreted the data. MMFvM wrote the manuscript, and all authors critically revised and commented on the manuscript. SGMAP supervised the study.

\section{Data Availability Statement}

The data that support the findings of this study are available from the corresponding author upon reasonable request.

\section{References}

1 Weidinger S, Beck LA, Bieber T, Kabashima $\mathrm{K}$, Irvine AD. Atopic dermatitis. Nat Rev Dis Primers. 2018;4(1):1.

2 Akdis CA, Akdis M, Bieber T, Bindslev-Jensen $C$, Boguniewicz M, Eigenmann $P$, et al. Diagnosis and treatment of atopic dermatitis in children and adults: European Academy of Allergology and Clinical Immunology/ American Academy of Allergy, Asthma and Immunology/PRACTALL Consensus Report. J Allergy Clin Immunol. 2006;118(1): 152-69.

3 Bin L, Leung DY. Genetic and epigenetic studies of atopic dermatitis. Allergy Asthma Clin Immunol. 2016;12:52.

4 Bjerre RD, Bandier J, Skov L, Engstrand L, Johansen JD. The role of the skin microbiome in atopic dermatitis: a systematic review. Br J Dermatol. 2017;177(5):1272-8.

5 Geoghegan JA, Irvine AD, Foster TJ. Staphylococcus aureus and Atopic Dermatitis: A Complex and Evolving Relationship. Trends Microbiol. 2017.

6 Travers JB. Toxic interaction between Th2 cytokines and Staphylococcus aureus in atopic dermatitis. J Invest Dermatol. 2014;134(8): 2069-71.

7 Clausen ML, Agner T, Lilje B, Edslev SM, Johannesen TB, Andersen PS. Association of Disease Severity With Skin Microbiome and Filaggrin Gene Mutations in Adult Atopic Dermatitis. JAMA Dermatol. 2018;154(3): 293-300.

8 Williams MR, Gallo RL. The role of the skin microbiome in atopic dermatitis. Curr Allergy Asthma Rep. 2015;15(11):65.

9 Williams MR, Nakatsuji T, Sanford JA, Vrbanac AF, Gallo RL. Staphylococcus aureus Induces Increased Serine Protease Activity in Keratinocytes. J Invest Dermatol. 2017; 137(2):377-84.

10 Nakatsuji T, Chen TH, Two AM, Chun KA, Narala S, Geha RS, et al. Staphylococcus aureus Exploits Epidermal Barrier Defects in Atopic Dermatitis to Trigger Cytokine Expression. J Invest Dermatol. 2016;136(11): 2192-200
11 Irvine AD, McLean WH, Leung DY. Filaggrin mutations associated with skin and allergic diseases. N Engl J Med. 2011;365(14):131527.

12 McAleer MA, Irvine AD. The multifunctional role of filaggrin in allergic skin disease. $\mathrm{J} \mathrm{Al-}$ lergy Clin Immunol. 2013;131(2):280-91.

13 Drislane $\mathrm{C}$, Irvine $\mathrm{AD}$. The role of filaggrin in atopic dermatitis and allergic disease. Ann Allergy Asthma Immunol. 2020;124(1):36-43.

14 Palmer CN, Irvine AD, Terron-Kwiatkowski A, Zhao Y, Liao H, Lee SP, et al. Common loss-of-function variants of the epidermal barrier protein filaggrin are a major predisposing factor for atopic dermatitis. Nat Genet. 2006;38(4):441-6.

15 van Leersum FS, Nagtzaam IF, van Oosterhoud CN, Ghesquiere SAI, Brandts R, Gostynski A, et al. Improving the diagnostic yield for filaggrin; concealed mutations in the Dutch population. J Allergy Clin Immunol. 2020;145(6):1704-6 e2.

16 Sandilands A, Terron-Kwiatkowski A, Hull PR, O'Regan GM, Clayton TH, Watson RM, et al. Comprehensive analysis of the gene encoding filaggrin uncovers prevalent and rare mutations in ichthyosis vulgaris and atopic eczema. Nat Genet. 2007;39(5):650-4.

17 Chen H, Common JE, Haines RL, Balakrishnan A, Brown SJ, Goh CS, et al. Wide spectrum of filaggrin-null mutations in atopic dermatitis highlights differences between Singaporean Chinese and European populations. Br J Dermatol. 2011;165(1):106-14.

18 Wong X, Denil S, Foo JN, Chen H, Tay ASL Haines RL, et al. Array-based sequencing of filaggrin gene for comprehensive detection of disease-associated variants. J Allergy Clin Immunol. 2018;141(2):814-6.

19 Eijkelenboom A, Kamping EJ, Kastner-van Raaij AW, Hendriks-Cornelissen SJ, Neveling K, Kuiper RP, et al. Reliable Next-Generation Sequencing of Formalin-Fixed, Paraffin-Embedded Tissue Using Single Molecule Tags. J Mol Diagn. 2016;18(6):851-63.

20 Miajlovic H, Fallon PG, Irvine AD, Foster TJ. Effect of filaggrin breakdown products on growth of and protein expression by Staphylococcus aureus. J Allergy Clin Immunol. 2010;126(6):1184-e3.

$21 \mathrm{Ml} \mathrm{C}$. Staphylococcus aureus colonization in atopic eczema and its association with filaggrin gene mutations. Br J Dermatol. 2017.

22 Simpson EL, Villarreal M, Jepson B, Rafaels N, David G, Hanifin J, et al. Atopic dermatitis Subjects Colonized with Staphylococcus aureus have a Distinct Phenotype and Endotype. J Invest Dermatol. 2018.

23 Berents TL, Carlsen KC, Mowinckel P, Skjerven $\mathrm{HO}$, Kvenshagen B, Rolfsjord LB, et al. Skin Barrier Function and Staphylococcus aureus Colonization in Vestibulum Nasi and Fauces in Healthy Infants and Infants with Eczema: A Population-Based Cohort Study. PLoS One. 2015;10(6):e0130145.

24 Fieten KB, Zijlstra WT, van Os-Medendorp $\mathrm{H}$, Meijer Y, Venema MU, Rijssenbeek-Nouwens L, et al. Comparing high altitude treatment with current best care in Dutch children with moderate to severe atopic dermatitis (and asthma): study protocol for a pragmatic randomized controlled trial (DAVOS trial). Trials. 2014;15:94

25 Fieten K, Schappin R, Zijlstra W, Figee L, Beutler J, Raymakers F, et al. Effectiveness of alpine climate treatment for children with difficult to treat atopic dermatitis: results of a pragmatic randomized controlled trial (DAVOS trial). Clin Exp Allergy. 2017.

26 van Mierlo MMF, Totte JEE, Fieten KB, van den Broek TJ, Schuren FHJ, Pardo LM, et al. The influence of treatment in alpine and moderate maritime climate on the composition of the skin microbiome in patients with difficult to treat atopic dermatitis. Clin Exp Allergy. 2019;49(11):1437-45.

27 Gonzáles I DS, Martin P.G.P et al. CCA: An R Package to extend canonical correlation analysis., 23. JoSS.

28 Oksanen J BF, Friendly M et al. The Vegan Package. Community Ecology Package. [2802-2018]; Available from: https:// cran.r-project.org/web/packages/vegan/vegan.pdf. 
29 Wickham H. ggplot2: Elegant Graphics for Data Analysis Springer-Verlag; 2009.

30 Harrison XA, Donaldson L, Correa-Cano ME, Evans J, Fisher DN, Goodwin CED, et al. A brief introduction to mixed effects modelling and multi-model inference in ecology. PeerJ. 2018;6:e4794.

31 Sandilands A, Sutherland C, Irvine AD, McLean WH. Filaggrin in the frontline: role in skin barrier function and disease. J Cell Sci. 2009;122(Pt 9):1285-94.

32 Morar N, Cookson WO, Harper JI, Moffatt MF. Filaggrin mutations in children with severe atopic dermatitis. J Invest Dermatol. 2007;127(7):1667-72.
33 Zeeuwen PL, Ederveen TH, van der Krieken DA, Niehues H, Boekhorst J, Kezic S, et al. Gram-positive anaerobe cocci are underrepresented in the microbiome of filaggrin-deficient human skin. J Allergy Clin Immunol. 2017;139(4):1368-71.

34 Feuillie C, Vitry P, McAleer MA, Kezic S, Irvine $\mathrm{AD}$, Geoghegan JA, et al. Adhesion of Staphylococcus aureus to Corneocytes from Atopic Dermatitis Patients Is Controlled by Natural Moisturizing Factor Levels. mBio. 2018;9(4).

35 Broccardo CJ, Mahaffey S, Schwarz J, Wruck L, David G, Schlievert PM, et al. Comparative proteomic profiling of patients with atopic dermatitis based on history of eczema herpeticum infection and Staphylococcus aureus colonization. J Allergy Clin Immunol. 2011; 127(1):186-93, 93 e1-11.
36 Simpson EL, Villarreal M, Jepson B, Rafaels N, David G, Hanifin J, et al. Patients with Atopic Dermatitis Colonized with Staphylococcus aureus Have a Distinct Phenotype and Endotype. J Invest Dermatol. 2018;138(10): 2224-33.

37 Oh J, Conlan S, Polley EC, Segre JA, Kong $\mathrm{HH}$. Shifts in human skin and nares microbiota of healthy children and adults. Genome Med. 2012;4(10):77.

38 Kong HH, Oh J, Deming C, Conlan S, Grice EA, Beatson MA, et al. Temporal shifts in the skin microbiome associated with disease flares and treatment in children with atopic dermatitis. Genome Res. 2012;22(5):850-9.

39 Grice EA, Segre JA. The skin microbiome. Nat Rev Microbiol. 2011;9(4):244-53. 